\title{
ACCURACY OF DEFECT DISTRIBUTIONS MEASURED BY BIAS DEPENDENT ADMITTANCE SPECTROSCOPY ON THIN FILM SOLAR CELLS
}

\author{
Decock K. ${ }^{1}$, Khelifi S. ${ }^{1}$, and Burgelman M. ${ }^{1}$ \\ ${ }^{1}$ Dept. of Electronics and Information Systems (ELIS), University of Gent, St-Pietersnieuwstraat 41, B-9000 Gent, Belgium \\ E-mail: Koen.Decock@elis.ugent.be
}

\begin{abstract}
Thin film solar cells have achieved efficiencies up to $20 \%$. Despite these excellent results, the understanding of the underlying mechanisms and the influence of defects on their performance is still incomplete. In thin film solar cells often defect level distributions are present rather than discrete defects. These distributions can be calculated from admittance measurements, however several assumptions are needed which hinder an exact defect density determination. By performing the measurements under different bias voltage conditions the accuracy of the method can be improved and assessed. This is illustrated with measurements on a flexible thin film $\mathrm{Cu}(\mathrm{In}, \mathrm{Ga}) \mathrm{Se}_{2}-$ based (CIGS) solar cell.
\end{abstract}

\section{INTRODUCTION}

Thin film solar cells have achieved efficiencies up to $20 \%$. Despite these excellent results, the understanding of the underlying mechanisms and the influence of defects on their performance is still incomplete. In thin film solar cells often defect level distributions are present rather than discrete defects. Walter et al. [1] developed a straightforward technique to determine defect density distributions from admittance measurements under zero bias conditions. This technique has recently been extended to non-zero bias conditions [2] which improves its applicability, enables to extract additional information from the measurements [3] and helps to improve and assess the accuracy of the results.

The abovementioned technique results in a graph of the defect density as a function of the trap energy with respect to conduction/valence band. This enables a fast interpretation of the results. Unfortunately several implicit assumptions have to be made when applying the theory which make the interpretation less straightforward. Additionally several (parameter) choices have an influence on the final result. The defect energy level (abscissa) can be determined in an accurate and objective way because the determination of the attempt to escape frequency is very straightforward. The determination of the defect density of states (ordinate) is more complicated. First of all, there is some arbitrariness in the choice of physical model for the band bending in the sample. Secondly, parameters such as the built-in voltage $\left(V_{b i}\right)$ and the position of the Fermi level in the bulk of the semiconductor $\left(E_{F n^{\infty}}\right)$ influence the final result. Performing the measurements at different bias voltages facilitates the determination of these parameters and models and allows to assess the accuracy of the final results.

\section{THEORY}

The technique developed by Walter et al. [1] extracts the defect density distribution as a function of the defect energy level from the derivative of the capacitance to the (logarithm of the) measurement frequency.

A defect level can only contribute to the capacitance up to a certain frequency which depends on the defect energy level position. This leads to a step in the capacitancefrequency $(C-f)$ characteristic and to an extremum in its derivative. As shown in [1] and [2], the defect energy level which corresponds to such an inflection frequency can be calculated as (1).

$$
E_{\omega}=k T \ln \left(\frac{\xi_{0} T^{2}}{\omega}\right)=k T \ln \left(\frac{2 v_{0}}{\omega}\right)
$$

Where $k$ represents Boltzmann's constant, $T$ the temperature, $\omega$ the angular frequency and $v_{0}$ the attempt to escape frequency. In order to cover a broad energy range, measurements at different temperatures have to be merged together using (1). The attempt to escape frequency can be determined either by plotting the angular frequency where the capacitance drops as a function of the inverse of the temperature (Arrhenius diagram) or by fitting its value in order that all defect distributions measured at various temperatures coincide in the overlapping region of their spectrum.

In order to calculate the defect density from the derivative of the capacitance an assumption on the band bending and the small signal Fermi level splitting has to be made. There are three main physical models: assuming linear band bending while assuming the local small signal Fermi level shift to be constant (2) or linear (3), or assuming parabolic band bending (4).

$$
\begin{gathered}
N_{t}\left(E_{\omega}\right)=-\frac{V_{b i}-V}{q w} \frac{d C}{d \omega} \frac{\omega}{k T} \\
N_{t}\left(E_{\omega}\right)=-\frac{\left(V_{b i}-V\right)^{2}}{w\left[q V_{b i}-E_{F n \infty}+E_{\omega}\right]} \frac{d C}{d \omega} \frac{\omega}{k T}
\end{gathered}
$$




\section{$37^{\text {th }}$ IEEE PVSC - Seattle 2011 - DOI: 10.1109/pvsc.2011.6186280}

$$
N_{t}\left(E_{\omega}\right)=-\frac{2\left(V_{b i}-V\right)^{3 / 2}}{w \sqrt{q} \sqrt{q V_{b i}-E_{F n \infty}+E_{\omega}}} \frac{d C}{d \omega} \frac{\omega}{k T}
$$

Where $N_{t}$ represents the defect density, $w$ the depletion width, $q$ the elementary charge and $C$ the capacitance. $V_{b i}$ is the built-in voltage and $V$ the applied bias voltage. $E_{F n^{\infty}}$ represents a Fermi level energy. The linear constant model (2) is the most straightforward (no appearance of $\left.E_{F n^{\infty}}\right)$. The linear model (3) is adequate for pin-junctions, the parabolic model (4) for most $n^{+} p$ junctions.

For a spatially constant defect density the derivative of the capacitance is proportional to $w \times\left(V_{b i}-V^{-1} \sim\left(V_{b i}-V^{-1 / 2}\right.\right.$ assuming the linear constant model. Proportional to $w \times\left(V_{b i}\right.$ $-V)^{-2} \sim\left(V_{b i}-V\right)^{-3 / 2}$ assuming the linear band bending, and assuming parabolic band bending it is proportional to $w \times\left(V_{b i}-V\right)^{-3 / 2} \sim\left(V_{b i}-V\right)^{-1}$.

Next to defect distributions, also other processes can cause a step in the $C$-f- characteristic [4], such as free carrier relaxation or the presence of a back contact barrier. Free carrier relaxation usually occurs at lower temperatures $(T<150 \mathrm{~K})$ and can often be ruled out as a cause of a measured capacitance step. The presence of a back contact barrier however has a similar influence on the admittance of a solar cell. For example the voltage dependence of the derivative of the capacitance [2] is similar as in (2) and (4): proportional with $\left(V_{b i}-V^{-1 / 2}\right.$ or $\left(V_{b i}-V^{-1}\right.$. Here we will assume the capacitance step to be caused by the presence of a defect distribution.

\section{EXPERIMENTAL}

Admittance measurements were performed on a flexible $\mathrm{Cu}(\mathrm{In}, \mathrm{Ga}) \mathrm{Se}_{2}$-based (CIGS) solar cell device $(n>15 \%)$ provided by EMPA. The sample was mounted in a $\mathrm{N}_{2}-$ coolable cryostat varying the temperature between 100 and $360 \mathrm{~K}$ in steps of $20 \mathrm{~K}$. The frequency was varied from $100 \mathrm{~Hz}$ to $10 \mathrm{MHz}$. The dc-bias voltage was varied between -2.0 and $0.6 \mathrm{~V}$. Data points which are hampered by parasitic network elements [5] or with an admittance phase angle smaller than $10^{\circ}$ have been discarded.

\section{RESULTS AND DISCUSSION}

A clear capacitance step was visible at forward voltage for temperatures in the range of $160-240 \mathrm{~K}$. Under different temperature/voltage conditions the capacitance is decreasing with increasing frequency without exhibiting a clear inflection point.

\section{The Energy scale}

If a clear step is visible and the derivative of the capacitance $-\omega \cdot d C / d \omega$ reaches a maximum, an Arrhenius diagram can be constructed in order to determine the attempt to escape frequency. In the studied samples this is only possible under forward bias conditions. The results are shown in Fig. 1.
Attempt to escape frequencies at $300 \mathrm{~K}$ of $2.31 \mathrm{Ghz}$ at $0.3 \mathrm{~V}$ and $373 \mathrm{Mhz}$ at $0.6 \mathrm{~V}$ are calculated. The attempt to escape frequency can also be determined by varying its value so that all defect distributions at various temperatures coincide in the overlapping region of their spectrum, as shown in Fig. 2. Starting from the attempt to escape frequency calculated from the Arrhenius diagrams, usually a good overlap can still be found when varying the attempt to escape frequency by a factor of 10 , leading to a difference in the calculated energy of $k \pi n 10 \approx 2.3 k T$. At $200 \mathrm{~K}$ this can lead to errors in the order of $40 \mathrm{meV}$.

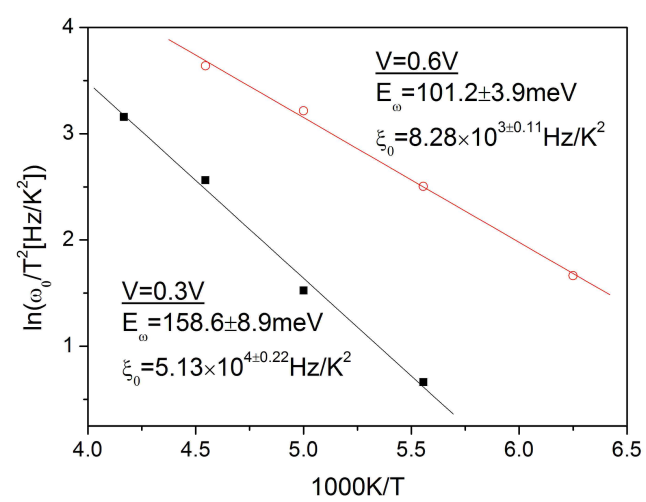

Figure 1: Arrhenius diagrams under forward bias conditions. The fitted parameter values are shown with $95 \%$ confidence intervals.

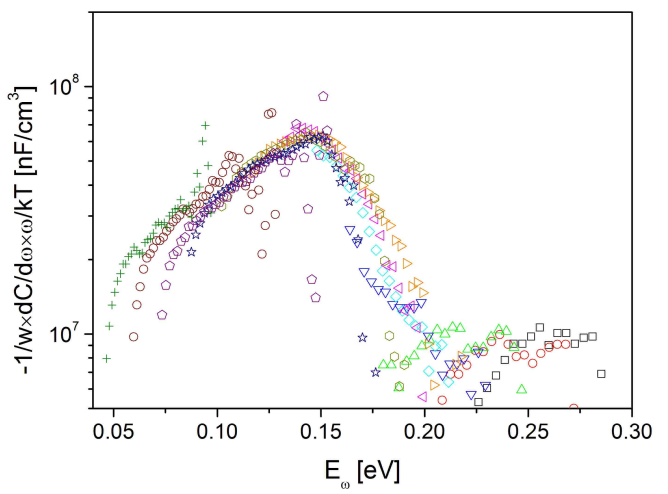

Figure 2: scaled derivative of the capacitance versus the energy level (1), calculated with $v_{0}=1 \mathrm{GHz}$ at $300 \mathrm{~K}$ $\left(\xi_{0}=22.2 \mathrm{kHz} / \mathrm{K}^{2}\right)$. Each symbol type/color represents a different temperature. Measurements performed under an applied bias of $0.3 \mathrm{~V}$.

If no clear maximum is present in the derivative of the capacitance at all, it can be hard to determine an adequate attempt to escape frequency and hence to set the energy scale. This is the case in the studied sample under reverse bias conditions. As it is possible to draw Arrhenius diagrams at forward voltage, we use a constant attempt to escape frequency of $1 \mathrm{GHz}$ at $300 \mathrm{~K}$ based on 


\section{$37^{\text {th }}$ IEEE PVSC - Seattle 2011 - DOI: 10.1109/pvsc.2011.6186280}

the results from these diagrams. In this case the attempt to escape frequency is not entirely correct for all applied voltages, but the procedure is very objective. In order to get to a more advanced determination of the energy scale, one can take into account the Meyer-Neldel type relation between the attempt to escape frequency and the activation energy [6]. This however requires an accurate determination of several energy vs. attempt to escape frequency pairs and the assumption that the capacitance drop connected with these pairs has the same origin.

\section{The density scale}

The factor $-\frac{1}{w} \frac{d C}{d \omega} \frac{\omega}{k T}$ is common in Eqs. (2), (3) and (4). It will be called the 'scaled derivative' and has units $\mathrm{nF} / \mathrm{cm}^{3}$. As can be seen in Fig. 2, a density spectrum determined from admittance measurements is a combined diagram of measurements at different temperatures. This is necessary because the useful frequency window for admittance spectroscopy is limited. The merged diagrams of the scaled derivative measured under different bias voltage conditions are shown in Fig. 3 .

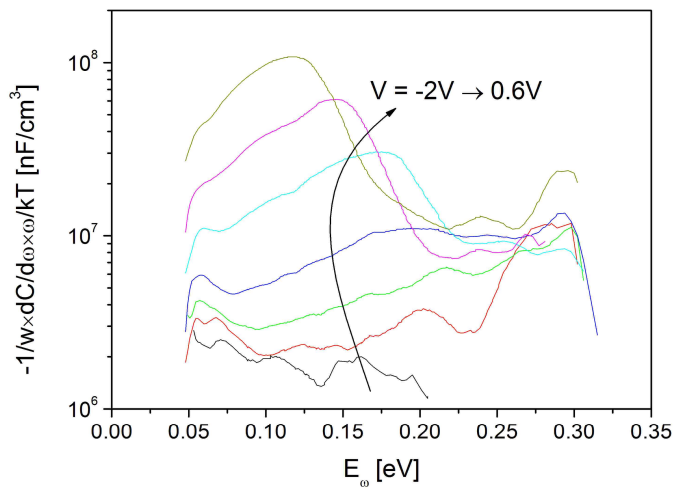

Figure 3: merged spectra of the scaled derivative at different bias voltages: $-2.0 \mathrm{~V} ;-1.5 \mathrm{~V} ;-1.0 \mathrm{~V} ;-0.5 \mathrm{~V} ; 0.0 \mathrm{~V}$; $0.3 \mathrm{~V}$ and $0.6 \mathrm{~V}$.

In order to scale the diagrams shown in Fig. 3 to get to a defect density according to Eqs. (2), (3) or (4) a value of $V_{b i}$ has to be obtained. When extracting the built-in voltage from CV measurements through Mott-Schottky analysis it is hard to arrive at an exact value of the built-in voltage because its value depends on the temperature at which the $C V$ measurement has been performed and on the part of the Mott-Schottky curve which is used to extrapolate to get to the intersection of with the abscissa. For the studied samples values of $V_{b i}$ in the order of $1-1.5 \mathrm{~V}$ are found at temperatures around $200 \mathrm{~K}$.

A second possibility to obtain an adequate value of $V_{b i}$ is to fit the results of Fig. 3 to the equations, assuming the measured defect density remains constant when varying the applied bias voltage. In order to be able to do this a defect energy level where the defect density can be assumed spatially uniform has to be selected. The most obvious choice in Fig. 3 is to choose a small energy value, e.g. $0.06 \mathrm{eV}$. if the assumption of spatial uniformity is wrong, it will be hard to make a good fit.

As it is not likely that the studied samples exhibit linear band bending ( $n^{+} p$-hetero junction), no fit will be made to Eq. (3). The properties $V_{b i}$ and $N_{t}$ are considered as parameters which are varied in order to get a good fit. $E_{F n \infty}$ in (4) however should not be varied as any variation in $E_{F n_{\infty}}$ can be counteracted with a variation in $N_{t}$ Considering an $n^{+} p$ hetero junction, $E_{F n_{\infty}}$ represents the position of the Fermi-level at the interface with respect to the valence band position of the $p$-type region. Due to the high $n$-type doping this level will be close to the conduction band, hence a good choice for $E_{F n_{\infty}}$ would be smaller than but close to the band gap value of the $p$-type region.

In Fig. 4 the values of the scaled derivative at $0.06 \mathrm{eV}$ at different bias voltages are shown together with the fits according to Eqs. (2) and (4). Table 1 shows the resulting parameters of the fitting procedure.

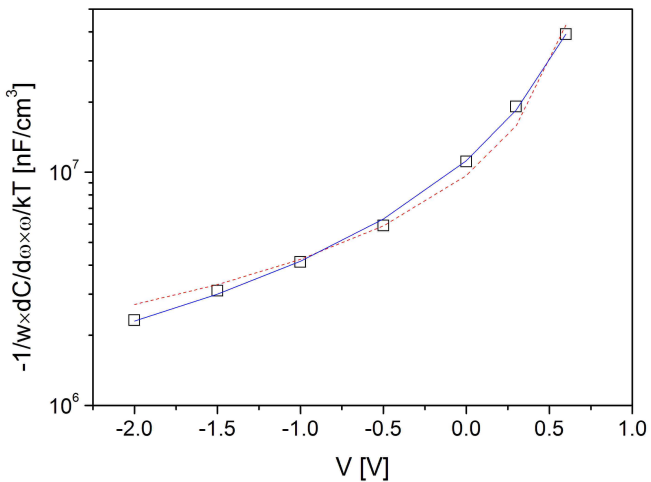

Figure 4: Fitting of the scaled derivative according to Eqs. (2) (red dashed line) and (4) (blue solid line). The choice of $E_{F n_{\infty}}$ has no influence on the resulting fitted characteristic when using Eq. (4).

\begin{tabular}{lcc}
\hline & $V_{b i}[\mathrm{~V}]$ & $N_{t}\left[\mathrm{~cm}^{-3} / \mathrm{eV}\right]$ \\
\hline Eq. (2) & $0.776_{ \pm} 0.084$ & $4.7 \times 10^{16_{ \pm} 0.087}$ \\
Eq. (4): $E_{F n_{\infty}=0.8 \mathrm{eV}}$ & $1.063_{ \pm} 0.043$ & $2.7 \times 10^{17_{ \pm} 0.017}$ \\
Eq. (4): $E_{F n_{\infty}}=0.9 \mathrm{eV}$ & $1.063_{ \pm} 0.043$ & $3.3 \times 10^{17_{ \pm} 0.024}$ \\
Eq. (4): $E_{F n_{\infty}}=1.0 \mathrm{eV}$ & $1.063_{ \pm} 0.043$ & $4.4 \times 10^{17_{ \pm} 0.054}$ \\
\hline
\end{tabular}

Table 1: Overview fitted parameters with $95 \%$ confidence intervals.

The best fit is found using Eq. (4). The value of $V_{b i}$ found in this case is in the region which is expected from $\mathrm{CV}$ measurements. The error bars on the fitted parameters are small, however, the choice of $E_{F n_{\infty}}$ has a substantial influence on the value of $N_{t}$ obtained. The choice of $E_{F n_{\infty}}$ does not only have an influence on the value of $N_{t}$ at $0.06 \mathrm{eV}$, but also on the ratio between $N_{t}$ calculated at low and high energy levels, through the factor in the nominator 


\section{$37^{\text {th }}$ IEEE PVSC - Seattle 2011 - DOI: 10.1109/pvsc.2011.6186280}

of Eq. (4). Taking into account the errors of the fitting procedure and the choice of $E_{F n_{\infty}}$, there is an uncertainty of a factor two on the value of $N_{t}$.

The fitted value of $V_{b i}$ can then be used to calculate the entire defect spectra as shown in Fig. 5 . The curves have been scaled in such a way that the defect density at $0.06 \mathrm{eV}$ is spatially uniform. The only justification for this is the goodness of fit in Fig. 4. Slight non uniformities can still lead to rather good fitting results, hence while assessing the results shown in Fig. 5, the assumptions whilst fitting should always be considered. When there are large non uniformities at a certain energy level however, it will be impossible to make a good fit. This is e.g. the case when performing the fitting procedure at $0.17 \mathrm{eV}$ in stead of at $0.06 \mathrm{eV}$ for the studied samples.

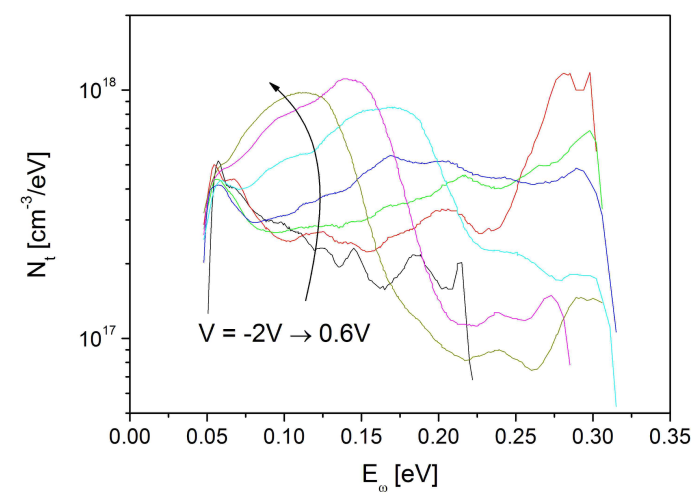

Figure 5: Resulting defect density calculated using Eq. (4) with $E_{F n_{\infty}}=0.9 \mathrm{eV}$. The same colors as in Fig. 3 have been used.

\section{CONCLUSIONS}

A procedure to determine the defect density profile of a thin film solar cell sample through the derivative of the capacitance has been assessed.

The most accurate way to determine the energy scale is through the drawing of an Arrhenius diagram. When this is not possible, measurement data at several temperatures have to be merged together. Performing measurements at different bias voltages can improve accuracy as sometimes Arrhenius diagrams can only be made in a certain bias voltage interval.

In order to determine the density scale, additional assumptions on the band bending, built-in voltage and Fermi level position are needed. Performing measurements at different bias voltages improves and helps to assess the accuracy of these assumptions. However an assumption on spatial uniformity is still needed at one energy level.

The defect density profile of a flexible thin film CIGS solar cell has been determined. The accuracy of the energy scale is estimated as $\pm 40 \mathrm{meV}$. The defect density is thought to be accurate up to a factor two.

\section{ACKNOWLEDGEMENTS}

We acknowledge the support of the Research Foundation - Flanders (K.D., FWO Ph.D. fellowship). We thank EMPA (CH) for providing CIGS samples.

\section{REFERENCES}

[1] T. Walter, et al., "Determination of defect distributions from admittance measurements and application to $\mathrm{Cu}(\ln , \mathrm{Ga}) \mathrm{Se}_{2}$ based heterojunctions," Journal of Applied Physics 80, pp. 4411-4420, Oct 1996.

[2] K. Decock, et al., "Defect distributions in thin film solar cells deduced from admittance measurements under different bias voltages," unpublished.

[3] J. Schafferhans, et al., "Oxygen doping of P3HT:PCBM blends: Influence on trap states, charge carrier mobility and solar cell performance," Organic Electronics 11, pp. 16931700, 2010.

[4] A. Niemegeers, et al., "Interpretation of capacitance spectra in the special case of novel thin film CdTe/CdS and CIGS/CdS solar cell device structures," in $2^{\text {nd }}$ World Conference on Photovoltaic Energy Conversion, Wien, 1998, pp. 1071-1074.

[5] J. H. Scofield, "Effects of series resistance and inductance on solar cell admittance measurements," Solar Energy Materials and Solar Cells 37, pp. 217-233, May 1995.

[6] A. Yelon, et al., "Multi-excitation entropy: its role in thermodynamics and kinetics," Reports on Progress in Physics 69, pp. 1145-1194, Apr 2006. 